\title{
X-RAY TOMOGRAPHY RECONSTRUCTION OF MULTIPHASE FLOWS AND THE VERIFICATION OF CFD
}

\author{
SANDY BLACK \& MARC LAING \\ TUV SUD NEL, East Kilbride, Scotland.
}

\begin{abstract}
Experimental studies using an X-ray tomography system were performed on a 4-inch horizontal section of the multiphase flow loop at NEL for gas-water and gas-oil-water flows. Values of liquid holdup and water liquid ratio are reported alongside analysis of the phase linear fraction through the crosssection of the pipe. The X-ray system revealed areas of gas entrainment and separation of oil and water which are not evident from high-speed video footage. The flow pattern of the tests was stratified-wavy, and computational fluid dynamics (CFD) analysis was performed using the volume of fluid (VOF) method. The prediction of liquid holdup and gas distribution through the pipe height as determined by CFD, correlated well with that determined by X-ray tomography. However, the results suggest that a transient VOF with a high-order mesh resolution is required to account for gas entrainment. This study shows that an X-ray system can be utilised to provide quantifiable validation data which are of value to multiphase models in CFD and provide insight that is not apparent during high-speed video analysis. The data generated from this system will be of considerable value to multiphase flow specialists and instrumentation developers.
\end{abstract}

Keywords: CFD, Multiphase, Void fraction, Tomography, X-Ray.

\section{INTRODUCTION}

Experimental measurements of multiphase flows have been extensively studied in the literature for horizontal pipes [1] and vertical pipes [2]. From this information, flow pattern or flow regime maps have been generated through empirical relationships or mechanistic models $[3,4]$ which are widely used today. For example, Mandhane et al. [4] identified six flow regimes in horizontal flow based on the superficial gas and liquid velocities, namely stratified, wavy, annular, bubble, slug and dispersed flow. The characteristics of these flow regimes may be simple, such as stratified flow where liquid flows at the bottom of the pipe and gas flows at the top or unsteady which can transition between regimes. An example would be slug flow where large gas bubbles are separated by pockets of liquid that fill the pipe, known as slugs. To determine these flow regimes, a typical approach is to use videos or photographs from laboratory test data. This approach can be highly subjective and more recently, sophisticated instrumentation such as high-speed video, gamma densitometry, electrical resistance probes and fast response X-ray tomography have been used to produce quantitative data. Subsequently, these data can be used to develop, verify, and validate models such as those used in computational fluid dynamics (CFD) with better accuracy [5].

The focus of this paper is to utilise an X-ray tomography system for the primary purpose of better understanding multiphase flows and verifying CFD simulations. The device reconstructs a 2D cross-sectional digital image of the flow field through computerised tomography (CT) and shows the distribution of gas, oil and water within the pipe. Moreover, the system delivers 'real-time' qualitative and quantitative data such as void fraction and water liquid ratio (WLR). Experimental measurements using the X-ray tomography system of gas-water and gas-water-oil flows were conducted in NEL's multiphase flow loop in a 4-inch horizontal section of pipework followed by multiphase CFD simulations. This approach is a significant improvement on the subjective nature of using a high-speed camera and an 
engineer to determine the flow regime and evaluate the limitations of the models. Utilising this technology allows verification of CFD models to a higher degree of confidence than with previous approaches.

A description of the X-ray system is given in Section 2 followed by the description of the test facility and selected experimental results in Sections 3 and 4, respectively. A comparison of CFD predictions against the X-ray measurements is given in Section 5 followed by conclusion in Section 6.

\section{X-RAY TOMOGRAPHY SYSTEM}

Within a multiphase flow, the gas, oil and water will typically attenuate an X-ray beam at different rates due to the different attenuation coefficients of the media. This measurement provides a means to indicate the flow pattern and structure of a multiphase flow through pipework provided the attenuation of the gas, oil and water is sufficiently different [6]. The use of $\mathrm{X}$-ray imaging can generally deliver measurements at higher sampling rates and spatial distribution compared to other non-invasive methods such as electrical capacitance tomography and gamma densitometry [6]. If more than one X-ray measurement is taken from different angles, the images can be numerically reconstructed to provide a computed tomography (CT) image. For the purposes of multiphase flow within a pipe, this would represent a 2-D cross section of the flow.

The REX-CELL ${ }^{\mathrm{TM}} 2 \mathrm{X}-\mathrm{RCN}$ system designed by FlowCapture ${ }^{\circledR}$ is a commercially available non-invasive measurement device that uses X-ray CT techniques to characterise gas-oil-water in multiphase flows and is to some extent analogous to that proposed by $\mathrm{Hu}$ et al. [6]. The system can be installed in non-metal circular pipes up to 6 inches in diameter and $16 \mathrm{~mm}$ in thickness at inclinations between $-90^{\circ}$ and $+90^{\circ}$. As shown in Fig. 1(a), the X-ray system consists of two independent sources and X-ray cameras which are oriented vertically and horizontally to the pipe. System operation, data acquisition and real-time data processing of the void fraction, liquid holdup and CT image is processed through a PC with the software, FlowDiary ${ }^{\mathrm{TM}}$. A collimator is used near the two X-ray sources to narrow the original cone-shaped beam to a rectangular beam. The X-ray cameras are positioned $140 \mathrm{~mm}$ from the centre of the pipe and are based on complementary metal-oxide-semiconductor

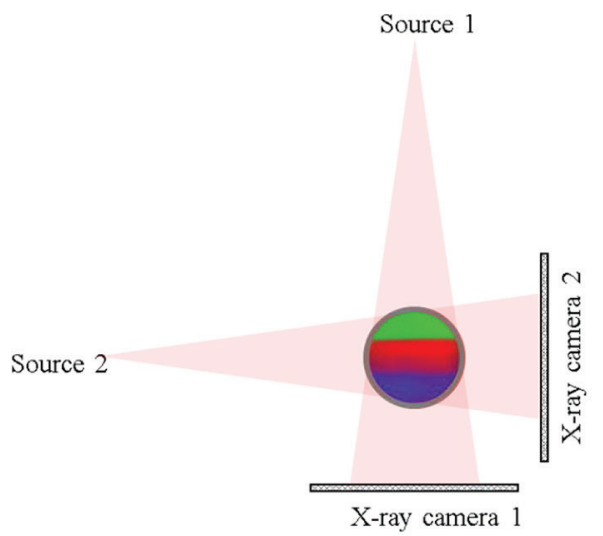

(a)

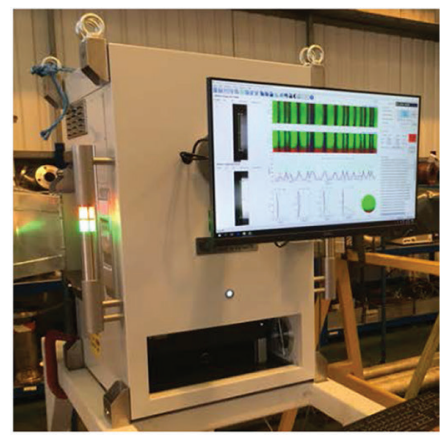

(b)

Figure 1: X-Ray tomography system: (a) schematic diagram, (b) installed system. 
image sensor technology with an effective pixel area of $3024 \times 864$ pixels $(74.8 \mu \mathrm{m} \times 74.8$ $\mu \mathrm{m}$ pixel size). At this resolution, a maximum of 60 frames per second (fps) can be achieved, however when $4 \times 4$ pixel binning is considered a maximum of $191 \mathrm{fps}$ can be achieved albeit with a reduced resolution of $768 \times 216$ pixels.

The system is installed over the pipe and is enclosed in lead shielding for safety as shown in Fig. 1(b). An X-ray must have sufficient power to penetrate the wall thickness, however at higher powers the attenuation of oil and water is similar which therefore becomes problematic when trying to distinguish between gas, oil and water in multiphase flows. Steel pipes typically have a higher attenuation than glass, Perspex or other composite pipes however cannot withstand higher pressures. For this reason, a fibre-reinforced thermoplastic polyether ether ketone (PEEK) carbon composite pipe was used which is rated to over 300 bar. The PEEK composite material is a commercially available alternative to traditional steelwork for the oil and gas industry with benefits in weight, erosion and corrosion resistance. The material provides the strength without compromising on wall thickness and is acquired for future experiments at pressures up to 140 bar in NEL's Advanced Multiphase Facility.

For all of the test conditions reported in this paper, the system was operated at $60 \mathrm{kV}$ and $3.3 \mathrm{~mA}$ with a sampling frequency of $40 \mathrm{fps}$. The key output of the X-ray device is the phase area fraction (PAF) of the gas, water and oil. Calibration of the device is achieved at these settings by filling the line with gas, followed by water and then oil at the same pressures and temperatures expected during the test programme.

A typical result for a gas-oil-water test is shown in Fig. 2. The instantaneous processed data over a 5 min test period from the X-ray is shown in Fig. 2(a) where the colours green, red and blue represent gas, oil and water, respectively. The top and middle graphs from top to bottom represent the instantaneous calculated fraction of gas, oil and water from the top and side X-ray cameras, respectively. At each instant, a CT image is produced as illustrated in Fig. 2(c) where various slugs and other flow structures are also captured. The 3D image in Fig. 2(c) is constructed from each of the CT images. At each sample time, a PAF of the gas, oil and water fraction can be computed which is illustrated in the bottom graph of Fig. 2(a). The average PAF can then be computed over the sample period therefore giving information

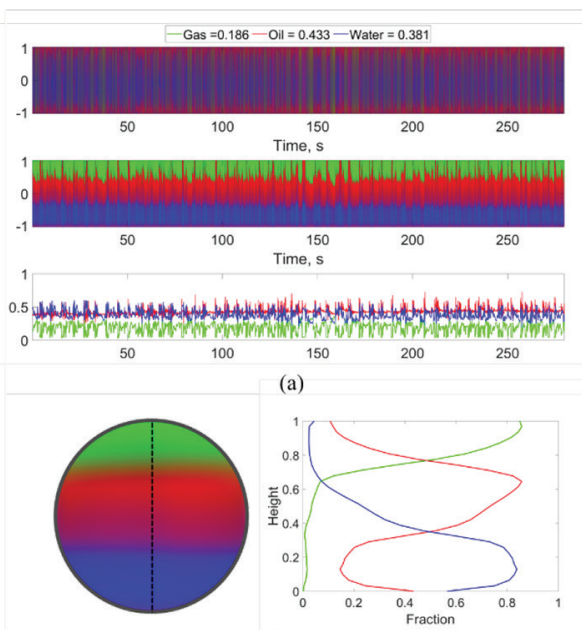

(b)

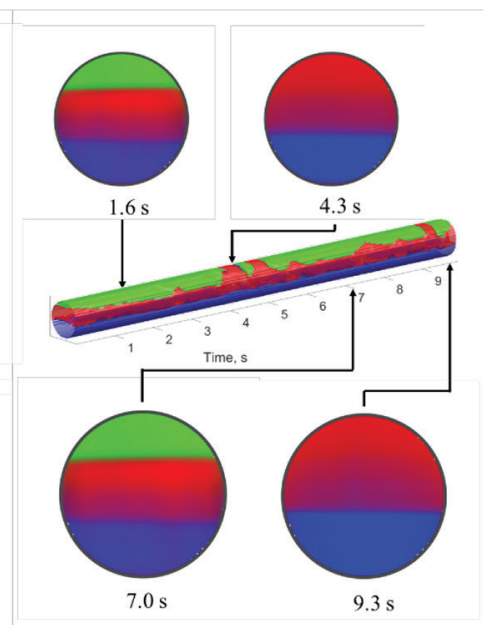

(c)

Figure 2: Typical output from X-ray device. 
on gas void fraction, liquid holdup and WLR. Additionally, information regarding the distribution through the pipe can be extracted from the CT plots, as shown in Fig. 2(b) where the gas, oil and water fractions are shown with respect to pipe height. Clearly, in this situation gas is at the top of the pipe whilst in the liquid layer at the bottom of the pipe, the lighter density oil is separated from the water.

\section{EXPERIMENTAL SETUP}

The NEL Multiphase Facility is based around a three-phase separator which contains the working bulk liquid. The oil and water are re-circulated around the test facility using two variable speed pumps and nitrogen is used as the gas phase and can deliver up to $0.5 \mathrm{~kg} / \mathrm{s}$ by evaporation of liquid nitrogen on demand. The delivery pressure of the nitrogen is up to 17 bar at the reference measurement location and after passing through the test section, nitrogen is exhausted to atmosphere from the separator.

The test section can accommodate test set-ups of lengths up to $60 \mathrm{~m}$ horizontal and $12 \mathrm{~m}$ vertical. Piping and adaptors are available to allow testing of 2, 3, 4 and 6-inch pipework and flow metres. The facility is manufactured entirely from stainless steel and is therefore capable of flowing brine substitutes and dead crude oils as the working fluids in addition to de-ionised water and refined oils. Perspex visualisation sections are also available in 2, 4 and 6-inch pipe sizes to allow for visual analysis of flow patterns which can be equipped with high-speed cameras to record the flow.

The test section used for the experimental test campaign and the corresponding dimensions are shown in Fig. 3:. The multiphase mixtures were introduced into a 2-inch Schedule 40 pipe and passed through a jet mixer to provide a uniform mixture condition at the inlet of the test section. The pipework was then orientated in a vertical loop of approximately $2 \mathrm{~m}$ in height. On the vertical pipework where the flow would be flowing downwards, the pipe size was increased to a 4-inch schedule 40 pipe (inner diameter of $102.26 \mathrm{~mm}$ ). After the vertical bend, a $2.75 \mathrm{~m}$ section of 4-inch schedule 40 pipe was positioned before a $1.05 \mathrm{~m}$ Perspex viewing section. A high-speed camera of $120 \mathrm{fps}$ was positioned at this location to provide visual flow patterns. A further $2 \mathrm{~m}$ of 4 -inch pipe was placed before a $1 \mathrm{~m}$ length section of 4 -inch PEEK pipe and the X-ray device was installed.

The fluids used in the test were nitrogen, a synthetic oil (Paraflex HT9) and an aqueous solution of magnesium sulphate and were typically performed at 10 bar. Typical properties of the gas, oil and water are given in Table 1 .

An extensive number of test points were recorded as part of research undertaken for the UK's National Measurement System Flow Programme [7]. A selection of these test points examined in this paper is shown in Table 2. Tests 155, 158 and 159 are gas-water tests which correspond to gas volume fractions (GVFs) of $73 \%, 51 \%$ and $23 \%$, respectively. Tests 208 ,

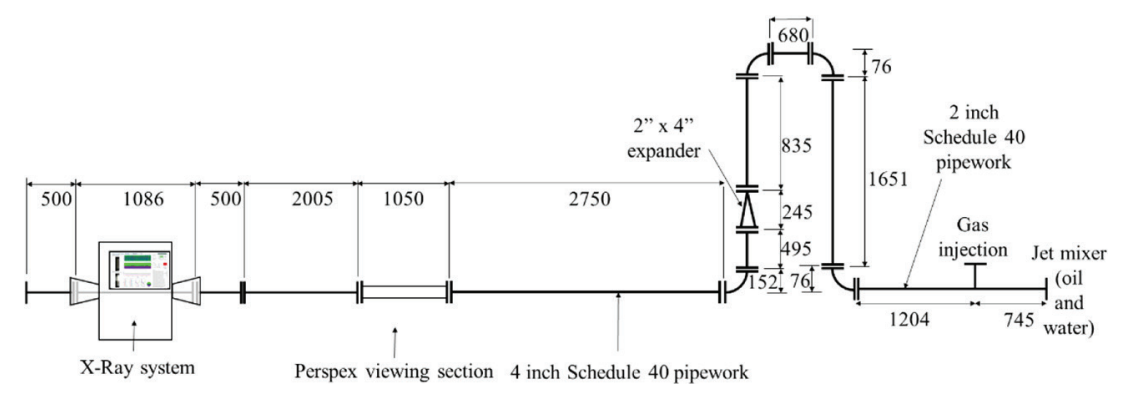

Figure 3: Line build. 
Table 1: NEL Fluid Properties $\left(20^{\circ} \mathrm{C}, 10 \mathrm{bar}(\mathrm{a})\right)$.

\begin{tabular}{llll}
\hline & Gas & Oil & Water \\
\hline Fluid & Nitrogen & Paraflex HT9 & $\mathrm{MgSO}_{4} 7 \mathrm{H}_{2} \mathrm{O}$ solution \\
Density $\left(\mathrm{kg} / \mathrm{m}^{3}\right)$ & 12.7 & 830.0 & 1050.0 \\
Viscosity $(\mathrm{cP})$ & $1.7710^{-2}$ & 16.00 & 1.30 \\
\hline
\end{tabular}

Table 2: Test matrix.

\begin{tabular}{|c|c|c|c|c|c|}
\hline \multirow[t]{2}{*}{ Test } & \multicolumn{3}{|c|}{ Flow rate $\left(\mathrm{m}^{3} / \mathrm{h}\right)$} & \multirow{2}{*}{$\begin{array}{c}\text { GVF } \\
\%\end{array}$} & \multirow{2}{*}{$\begin{array}{c}\text { WLR } \\
\%\end{array}$} \\
\hline & Gas & Oil & Water & & \\
\hline 155 & 41.72 & - & 8.76 & 82.6 & 100 \\
\hline 158 & 25.17 & - & 23.86 & 51.3 & 100 \\
\hline 159 & 10.70 & - & 35.61 & 23.0 & 100 \\
\hline 208 & 42.06 & 4.51 & 4.34 & 82.6 & 49.0 \\
\hline 205 & 23.43 & 11.36 & 11.44 & 50.6 & 50.2 \\
\hline 218 & 10.82 & 24.86 & 10.95 & 23.2 & 30.6 \\
\hline
\end{tabular}

205 and 218 correspond to gas-water-oil tests with GVF ratios of $83 \%, 51 \%$ and $23 \%$, respectively with WLR of $49 \%, 50 \%, 30.6 \%$.

\section{EXPERIMENTAL RESULTS}

\subsection{Absolute error}

To assess the absolute error of the X-ray tomography system, non-flowing (i.e. static) tests were performed. A known volume of fluid (VOF) was placed into a 3-inch sealed test section and placed into the X-ray system where a comparison between the absolute phase area average fractions of gas, oil and water was assessed. The absolute error of the system was shown by Black [7] to be within 3\% and 5\% for gas-liquid and gas-oil-water tests, respectively, which agrees with similar X-ray systems [6].

\subsection{Two-phase results}

Two-phase results of gas-water mixtures from the X-ray tomography system and snapshots from high speed videos are presented in Fig. 4 for tests 155, 158 and 159 which correspond to GVFs of $23 \%, 50 \%, 83 \%$, respectively. The corresponding average PAFs over each $1 \mathrm{~min}$ test are shown in Table 3.

Fig. 4 clearly shows that the liquid holdup from the videos correlates well with the level predicted by the X-ray. For Test 155 (23\% GVF), the video observation shows the flow is stratified-wavy (Fig. 4(a)) and this is captured by the small amplitude peaks in the X-ray output (Fig. 4(b)). The videos in Fig. 4(c)-(e) show that for higher GVF values of 52\% and $83 \%$, the waves hit the top of the pipe, with this occurring more frequently for the higher GVF test (Test 159). The output from the X-ray shows a drop in the gas content around $25 \mathrm{~s}$ 


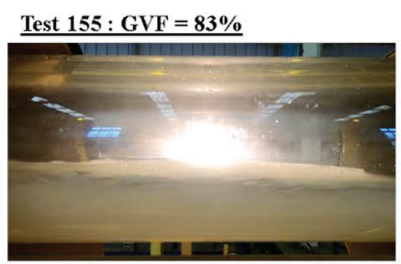

(a)

Test 158: GVF $=\mathbf{5 1 \%}$

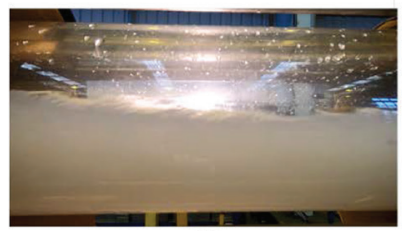

(c)

Test 159: GVF $=23 \%$

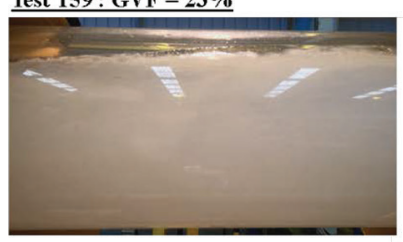

(e)

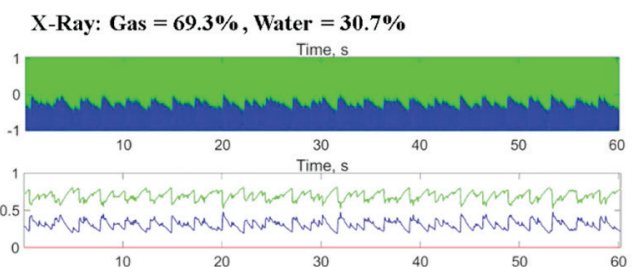

(b)

X-Ray: Gas $=\mathbf{4 2 . 7 \%}, \underset{\text { Water }}{=} \mathbf{5 7} . \mathbf{3} \%$

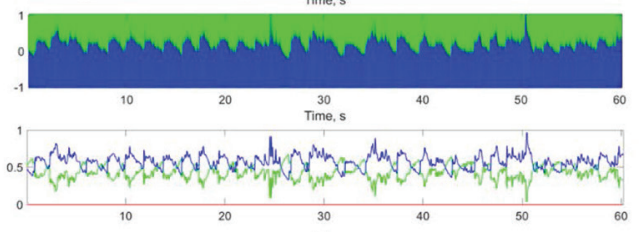

(d)

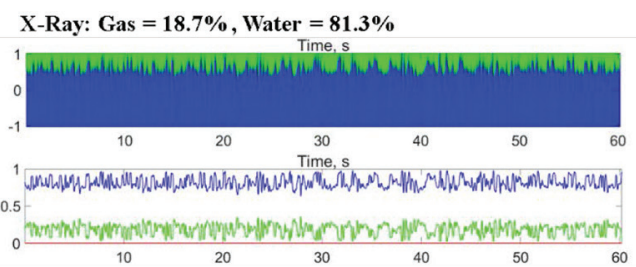

(f)

Figure 4: Two-phase gas water X-ray measurements and photographs for GVF of (a and b) $83 \%$, (c and d) $50 \%$, (e and f) $23 \%$.

Table 3: PAF results for gas, oil and water for the multiphase tests.

\begin{tabular}{ccccccc}
\hline Test & \multicolumn{3}{c}{ X-ray PAF } & \multicolumn{4}{c}{ Superficial velocities (m/s) } \\
& Gas & Oil & Water & Gas & Oil & Water \\
\hline 155 & 69.3 & - & 30.7 & 1.41 & - & 0.30 \\
158 & 42.7 & - & 57.3 & 0.36 & - & 1.20 \\
159 & 18.7 & - & 81.3 & 0.85 & - & 0.81 \\
208 & 53.5 & 28.8 & 17.8 & 1.42 & 0.15 & 0.15 \\
205 & 36.9 & 34.1 & 29.0 & 0.79 & 0.39 & 0.38 \\
218 & 17.0 & 54.9 & 28.1 & 0.37 & 0.37 & 0.84 \\
\hline
\end{tabular}

and 50 s for Test 158 (Fig. 4(d)), whereas this occurs regularly in Test 159 (Fig. 4(f)) due to the high VOF in the pipe.

The GVF in Table 2 is calculated from reference flow metres which are located approximately 600 pipe diameters upstream of the X-ray device. Experimental tests are run for a sufficient period to allow the flow to move completely through the system before flow rates are sampled. Due to the slip between the gas and the liquid, local liquid holdup can occur within the test section. Comparing the GVF in Table 2 with the gas void fraction measured by the X-ray in Table 3, the values from the X-ray are lower therefore giving a holdup of liquid within this section. To quantify the degree of slip, the slip ratio, $S$, can be used: 


$$
S=U_{G}\left(1-\varepsilon_{G}\right) / U_{L} \varepsilon_{G}
$$

where $U_{G}$ and $U_{L}$ are the superficial velocities of the gas and liquid based on a 4-inch pipe (I.D. $102.26 \mathrm{~mm}$ ), respectively, as given in Table 3 and $\varepsilon_{G}$ is the gas void fraction measured by the X-ray. The slip ratio is calculated as $2.1,1.4$ and 1.3 for Tests 155,158 and 159 which correspond to decreasing liquid-hold ups for the corresponding tests, i.e. the difference between the GVF measured at the reference meters and the X-ray are 13\%, $9 \%$ and $4 \%$ for Tests 155,158 and 159 , respectively.

The corresponding distribution of gas and liquid over the height of the pipe is shown in Fig. 5. An interesting observation is made where the liquid at the bottom of the pipe shows that some gas entrainment has occurred. As discussed by Hu et al. [6], gas entrainment is a strong function of liquid and gas flow rates. Upstream of the X-ray system, the gas enters at a $90^{\circ}$ angle to the water which has passed through a jet-mixer before being carried through a vertical U-bend. During this period, it is likely that a high degree of turbulent mixing of the gas into the liquid would occur, more notable for higher gas flow rates which is evident in Fig. 5.

\subsection{Gas-oil-water results}

There is a significant volume of work related to two-phase slug flows. However only a limited number of publications on three-phase X-ray results [6,7] are available. Gas-oil-water results from the X-ray and snapshots from high-speed videos are presented in Fig. 6 for Tests 208, 205 and 218 with GVF of 83\%, 51\% and 23\%, respectively, with WLR of 49\%, 50\%, 30.6\%. The corresponding average PAFs over each 1 min test are shown in Table 3.

Fig. 6 clearly shows similar trends compared to the two-phase results with similar GVF ratios where a stratified-wavy flow pattern is present in the videos and predicted by the X-ray. Small amplitude peaks are present which touch the top of the pipe more frequently as the GVF ratio is lowered. From the videos it is not clear if the water and oil are well mixed or have separated to some degree.

Comparing the GVF calculated from the reference flow metres in Table 2 against the gas PAF from the X-ray shows larger differences for higher GVF ratios of 29\%, $14 \%$ and $6 \%$ for GVF of $83 \%, 51 \%$, and $23 \%$ (i.e. Test 208, 205 and 218, respectively). Using the reference meter flow rates and assuming water and oil and uniformly mixed, the slip ratio is 4.1, 1.8 and 1.5 which relates to the trend of liquid holdup observed by the X-ray.

The time-average phase linear fraction from the X-ray is shown in Fig. 7 and shows that the distribution of gas (green) oil (red) and water (blue). As the data are averaged over the test period, the gas fraction is not equal to 1 in Test 205 and 218 (Fig. 7(b) and (c)) due to waves

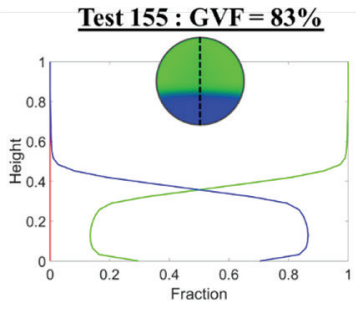

(a)

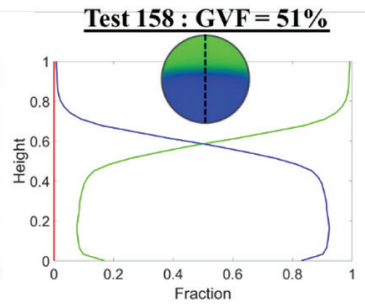

(b)

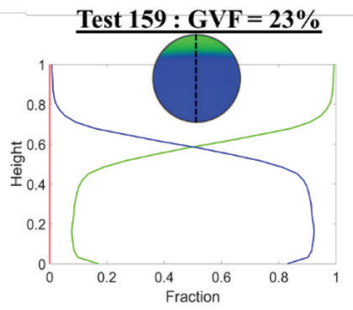

(c)

Figure 5: Two-phase gas water X-ray measurements over the height of the pipe for (a) Test 155, (b) Test 158 and (c) Test 159. 


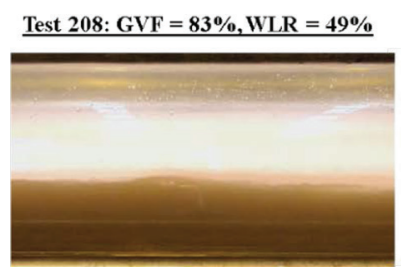

(a)
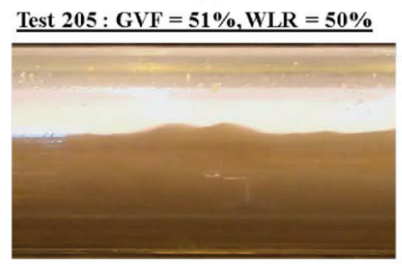

(c)

$\underline{\text { Test } 218: \text { GVF }=23 \%, \text { WLR }=31 \%}$

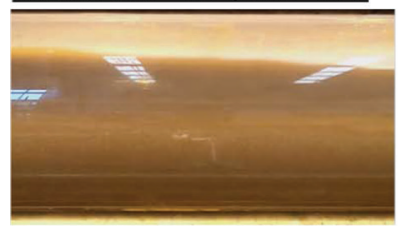

(e)

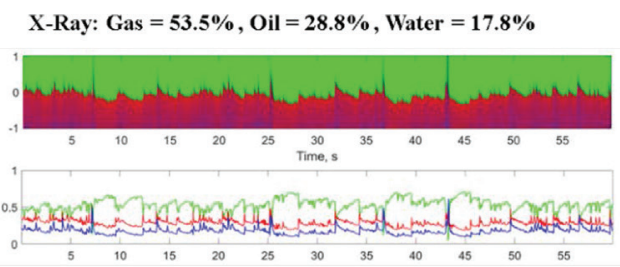

(b)

X-Ray: Gas $=36.9 \%$, Oil $=34.1 \%$, Water $=29.0 \%$

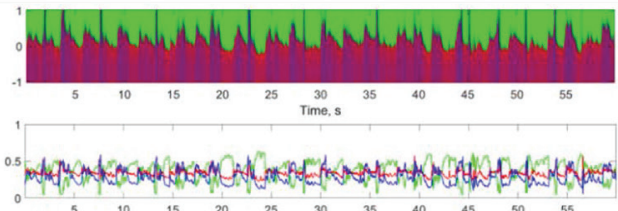

(d)

X-Ray: Gas $=\mathbf{1 7 . 0} \%$, Oil $=\mathbf{5 4 . 9} \%$, Water $=\mathbf{2 8 . 1} \%$

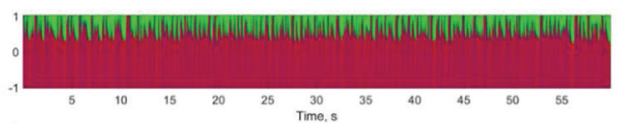

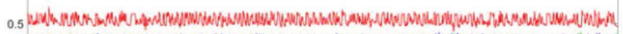

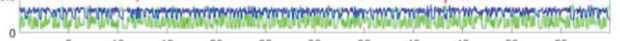

(f)

Figure 6: Gas-oil-water X-ray measurements and photographs for (a) Test 208; (b) Test 205 and (c) Test 218.

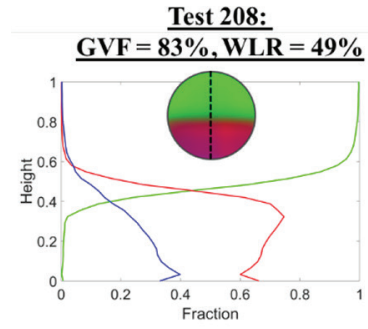

(a)

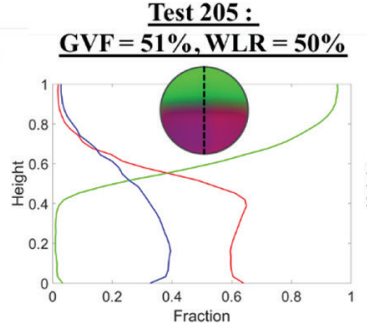

(b)

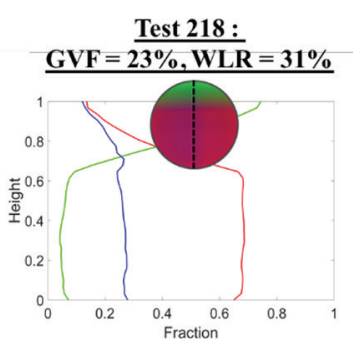

(c)

Figure 7: Gas-oil-water X-ray measurements over the height of the pipe for (a) Test 208; (b) Test 205 and (c) Test 218.

hitting the top of the pipe as shown by the small amplitude fluctuations in Fig. 6(d) and (f). The distribution of oil and water at the bottom of the pipe is only shown to be uniform in Test 218 (Fig. 7(c)). Peaks of oil are shown near the centre of the pipe in Fig. 7(a) and (b) for Test 208 and 205. This is due to the separation of the water and oil as the effects of gravity and surface tension dominate over the momentum of the flow. From the flow rates in Table 2, it is clear that Test 218 has the highest flow rates and therefore the X-ray results suggest sufficient turbulent mixing is still present, as shown by the uniform distribution in the liquid layer in Fig. 7(c). 


\section{CFD MODEL}

\subsection{Set-up}

The commercial CFD software, ANSYS Fluent 19.1 with the VOF approach [8] was used to model the interaction between the gas and the liquid with a sharp/dispersed interface scheme. The VOF method assumes that the fluids are immiscible and solves a single set of momentum equations where a volume fraction for each fluid is transported through the domain. The VOF method is a common approach for multiphase flow CFD and has been evaluated against flow pattern maps and various experimental measurements and observations [9,10]. Surface tension was also included with the continuum surface force model [11] and constant values of 72 and 34 dynes/cm for gas-water/oil interfaces for oil-water mixtures was used, respectively. The effects of wall adhesion were not considered.

\subsection{Geometry and mesh}

A three-dimensional geometry was used which represented a segment of the test facility. The geometry consisted of two separate 2-inch inlets for the gas and liquid with a vertical ' $U$ ' bend which expands into a 4-inch section. The position of the X-ray on the model was the same as the experimental location. Initially, two fully structured meshes were evaluated for Test 155 , namely mesh 1 and mesh 2 consisting of 1.6 million cells with an average cell size of $4.3 \mathrm{~mm}$ and 3.0 million cells with an average cell size of $3.5 \mathrm{~mm}$, respectively. Mesh 1 and 2 predicted a liquid hold-up $69.4 \%$ and $68.9 \%$, respectively. As the liquid hold-up values are with $5 \%$, which is the limit of the X-ray equipment, mesh 1 was used for the remaining simulations.

\subsection{Solution procedure}

An initial transient simulation was performed for Test 155 which showed transient effects in the vertical U-bend but these were dampened by the location of the X-ray device. Instead a steady-state solution was used. This is also preferred as this method can be applied more readily to the near industrial-scale simulations. The tracking of the interface was solved using an implicit time discretisation and a coupled algorithm with a first-order upwind scheme for momentum and compressive scheme for the volume fraction was used. The k- $\omega$ SST model with turbulence damping coefficient of 10 was used for turbulence.

\subsection{CFD results}

\subsubsection{Two-phase results}

Fig. 8(a), (b), (c) shows Tests 155, 158 and 159, respectively. A comparison between the time-averaged X-ray CT image and the steady-state CFD analysis for all of the cases in Fig. 8 compare well and the phase averaged values are within $4 \%$ as shown in Table 4 . As discussed previously, Fig. 8 shows a degree of gas and liquid entrainment in each test which is not accounted for in the CFD model as the VOF method inherently assumes that the two fluids are immiscible. Despite this assumption, the liquid levels agree relatively well. The CFD analysis typically has a sharper gradient along the height of pipe which is due to the steadystate approximation and will not replicate a wavy stratified flow. 


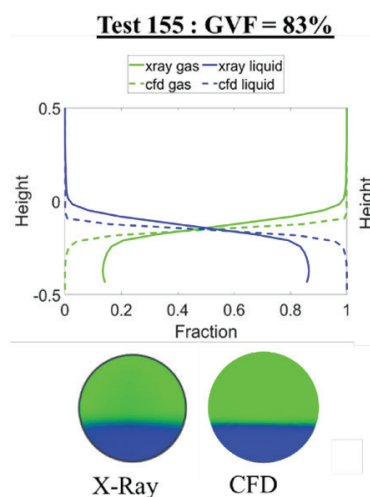

(a)

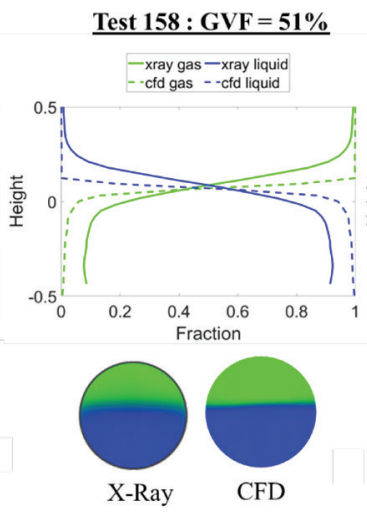

(b)
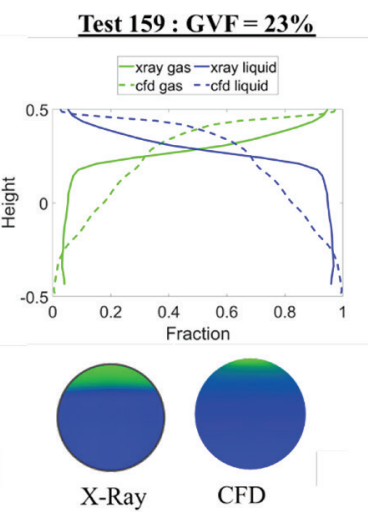

(c)

Figure 8: Results for Test case 155 showing ( $\mathrm{a}$ and $\mathrm{b}$ ) X-ray results and (b) CFD results.

Table 4: Absolute error between the X-ray and CFD simulations.

\begin{tabular}{cccc}
\hline Test & \multicolumn{3}{c}{ Absolute difference (\%) } \\
& Gas & Oil & Water \\
\hline 155 & 0.1 & & -0.1 \\
158 & -3.7 & & 3.7 \\
159 & 1.3 & & -1.3 \\
208 & 0.3 & -5.2 & 4.8 \\
205 & -7.1 & 0.9 & 6.2 \\
218 & 1.3 & 1.8 & -3.1 \\
\hline
\end{tabular}

\subsubsection{Three-phase results}

Fig. 9 shows the comparison between the X-ray measurements and the CFD simulations for Tests 208, 205 and 218. The prediction from the VOF model matches the output from the $\mathrm{X}$-ray relatively well as shown by comparing the X-ray CT image and cross-sectional from the CFD simulation in Fig. 9. Slight differences occur in the linear phase area gas fractions in Fig. 9 in the upper half of the pipe due to the wavy nature of the flow and the steady-state CFD analysis which only represents an ensemble of realisations (i.e. Reynolds averaging). It is important to reiterate that the inlet to the model is the exit of a jet mixer and it is assumed that the oil and water are well mixed. From the video evidence, it is suggested that at this point, the water and oil are well mixed; however, the X-ray shows otherwise. Relying on video evidence would lead to the choice of a steady-state implicit VOF approach. However, it is known to not be accurate when surface tension effects are important and is naturally diffusive. When the oil and water remain well mixed, this method is suitable for some gasoil-water flows such as Test 218 (Fig. 9(c)), however this is inappropriate when the phases separate further downstream of the inlet. 


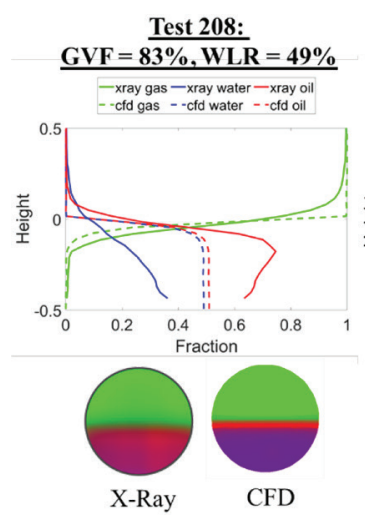

(a)

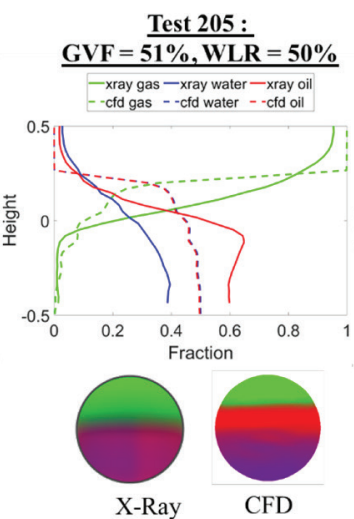

(b)

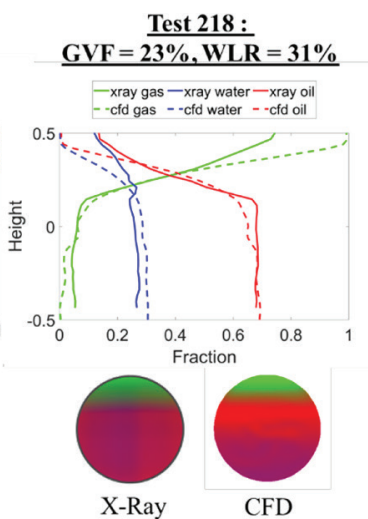

(c)

Figure 9: Results for Test case 218 showing (a and b) X-ray results and (c and d) CFD results.

\section{CONCLUSION}

Performing more detailed measurements of complex multiphase flows are of vital importance in aiding the understanding of multiphase flow model development. This study shows that an $\mathrm{X}$-ray system can be utilised to provide quantifiable validation data which is of value to multiphase models in CFD and provide insight that is not apparent during high-speed video analysis. Measurements using an X-ray system were conducted on a 4-inch horizontal section of NEL's multiphase flow facility for nitrogen-water and nitrogen-oil-water flows where values of liquid holdup and water liquid ratio were examined. This also included the analysis of phase linear fraction through the cross-section of the pipe to reveal areas of gas entrainment in the liquid and the initial separation of oil and water within oil-water flows. The VOF was examined to compare against the X-ray measurement and adequately captured the gas void fraction for the test cases examined. The model was not capable of predicting regions of gas entrainment and oil and water separation as captured by the X-ray system. Further model investigations should include a transient $\mathrm{VOF}$ with a high-order mesh resolution to attempt to capture gas entrainment.

The analysis generated from the X-ray tomography device will help resolve the limitation in current CFD modelling techniques of multiphase flows and will be of considerable value to multiphase flow specialists and instrumentation developers.

\section{ACKNOWLEDGEMENTS}

The work described in this report was carried out by TUV SUD Limited trading as NEL under contract to the Department of Business, Energy \& Industrial Strategy as part of the National Measurement System's Flow Programme. It was part funded by the Flow Programme and part funded by an Industrial Strategy Challenge Fund award.

\section{REFERENCES}

[1] Hall, A.R.W., Flow Patterns in Horizontal Three-Phase Flows of Oil, Water and Gas. Proceedings of Multiphase 1997, 1997.

[2] Beggs, D.H. \& Brill, J.P., A Study of Two-Phase Flow in Inclined Pipes. Journal of Petroleum Technology, 25(5), pp. 607-617, 1973. 
[3] Baker, O., Simultaneous Flow of Oil and Gas. Oil and Gas Journal, 53, pp. 185-190, 1954.

[4] Mandhane, J.M., Gregory, G.A. \& Aziz, K., A Flow Pattern Map for Gas-Liquid Flow in Horizontal Pipes. International Journal of Multiphase Flow, 1, pp. 537-553, 1974.

[5] Hill, T.J., Research in multiphase flow developed into new global discipline of flow assurance-an oil industry perspective. Proceedings of the 11th North American Conference on Multiphase Technology, 2018.

[6] Hu, B., Langsholt, M., Liu, L., Andersson, P. \& Lawrence, C., Flow structure and phase distribution in stratified and slug flows measured by X-ray tomography. International Journal of Multiphase Flow, 67, pp. 162-179, 2014.

[7] Black, S., Commissioning and Initial Performance of an X-ray tomography Device, NEL Report Number 2018/559, 2019.

[8] Hirt, C.W. \& Nichols, B.D., Volume of Fluid (VOF) method for the dynamics of free boundaries. Journal of Computational Physics, 39(1), pp. 201-225, 1981.

[9] De Schepper, S.C.K., Heynderickx, G.J. \& Marin, G.B., CFD modelling of all gas-liquid and vapor-liquid flow regimes predicted by the Baker chart. Chemical Engineering Journal, 138(1-3), pp. 349-357, 2008.

[10] Parvareh, A., Rahimi, M., Alizadehdakhel, A. \& Alsairfai, A.A., CFD and ERT investigations on two-phase flow regimes in vertical and horizontal tubes. International Communications in Heat and Mass Transfer, 37(1), pp. 304-311, 2010.

[11] Brackbill, J.U., Kothe, D.B. \& Zemach, C., A Continuum Method for Modelling Surface Tension. Journal of Computational Physics, 100(1), pp. 335-354,1992. 